4-1-2021

\title{
Karfafa neman maganin zazzabi da kulawa ga yara a tsakanin matan jihar Kebbi
}

Breakthrough RESEARCH

Follow this and additional works at: https://knowledgecommons.popcouncil.org/departments_sbsr-rh How does access to this work benefit you? Let us know!

\section{Recommended Citation}

Breakthrough RESEARCH. 2021. "Karfafa neman maganin zazzabi da kulawa ga yara a tsakanin matan jihar Kebbi," infographic. Washington, DC: Population Council. 


\section{Karfafa neman maganin zazzabi da kulawa ga yara a tsakanin matan jihar Kebbi}

Aikin Breakthrough RESEARCH ya gudanar da bincike a kan dabi'un mata game da kula da lafiya a lokacin da suka sami juna-biyu ko suka haihu a cikin shakaru biyu da suka wuce.

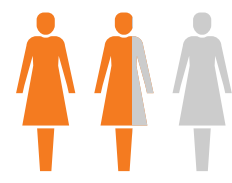

Kusan mata biyu (2) cikin ukun (3) (59\%) mata a jihar Kebbi su ka nemi magani da kulawa daga ma'akacin lafiya wa yaran su kasa da shekara biyu da suka kamu da zazzabi.

\section{Sakamakon Nazarin Jihar Kebbi}

Nazarin lura da halayyar ya gano muhimman abubuwa guda uku don inganta neman magani da kulawa daga ma'aikacin lafiya ga yara masu fama da zazzabi. Kashe-kashen da aka nuna a nan su na kasa da tsaka-tsakin makin na duk kananan hukumomn da aka zaba domin binciken. Matsakaitan adadin za su iya shan bambam da matsaikacin maki da aka nuna a shafi na gaba.

\section{Mata sun yarda cewa babu wanda ya fi cancanta a tuntuba a lokacin da yaro ya kamu da rashin lafiya illa ma'aikacin Iafiva}

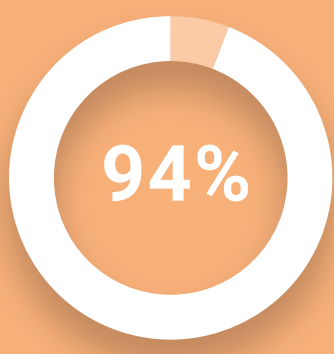

Kusan duka mata sun yarda cewa ya dace a tuntubi ma'aikacin lafiya duk lokacin da yaro bai da lafiya.
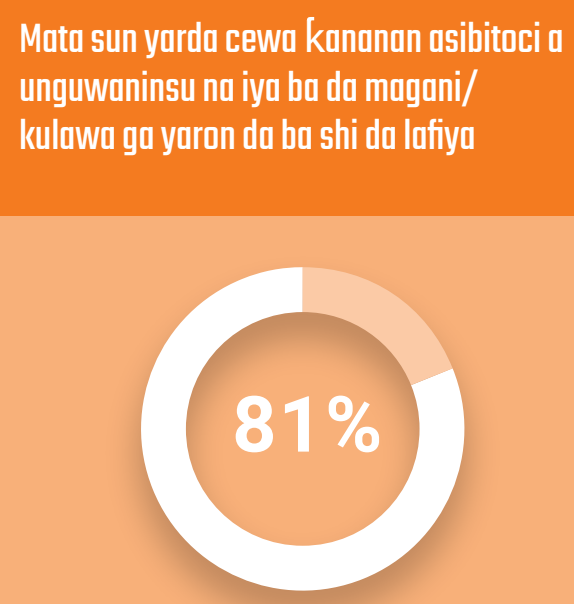

Mata hudu cikin biyar sun yarda cewa kananan asibitocin ungwaninsu na da magani da kuma iya kulawa da yaro duk lokacin da.
Mata suna da tabbaci akan samun izinin mazajen su akan neman shawara ko magani idan yara bai da lafiya

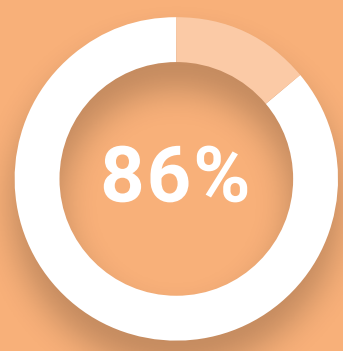

Mata hudu cikin biyar suna da tabbaci akan samun izinin mazajen su akan neman shawara ko magani idan yaro bai da lafiya.

\section{Idan an kara inganta waddanan abubuwan, neman magani zai iya karuwa a jihar Kebbi.}

\section{Sakamakon Nazari Na Kananan Hukumomi a Jihar Kebbi}

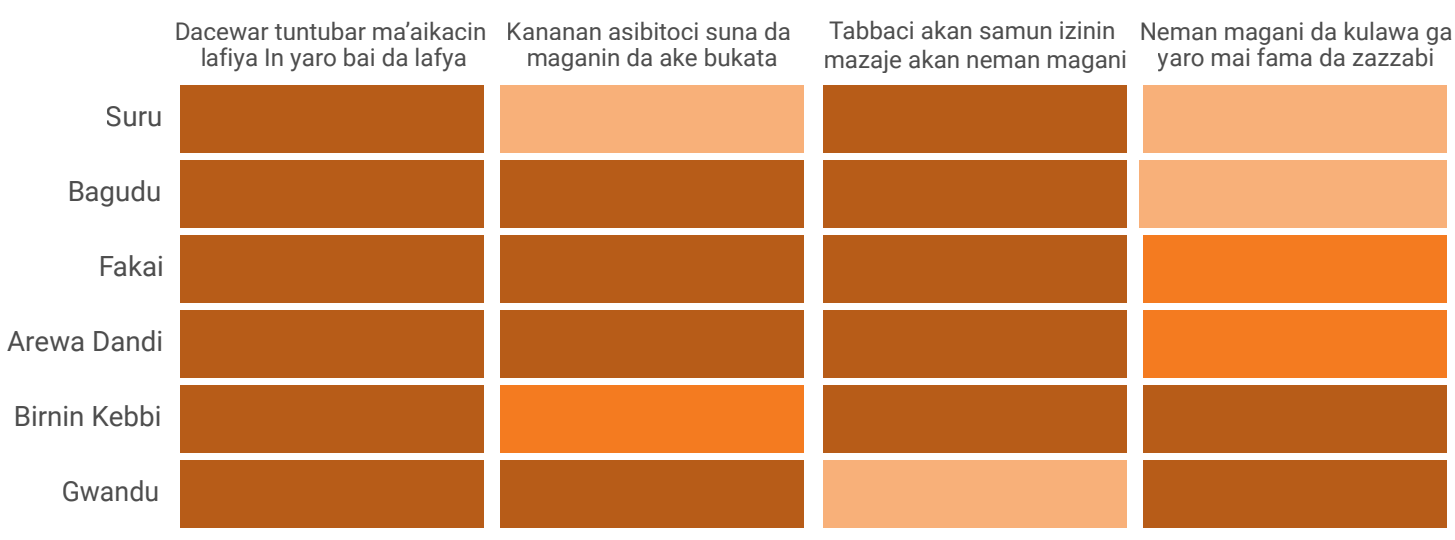

Yawan mata a cikin kashi dari da suka amsa da tabbaci ta kananan hukumomi

\section{$0 \%-24 \%$}

$25 \%-49 \%$

$50 \%-74 \%$

$75-100 \%$ 


\section{Karfafa neman maganin zazzabi da kulawa ga yara a tsakanin matan jihar Kebbi}

Aikin Breakthrough RESEARCH ya gudanar da bincike a kan dabi'un mata game da kula da lafiya a lokacin da suka sami juna-biyu ko suka haihu a cikin shakaru biyu da suka wuce.

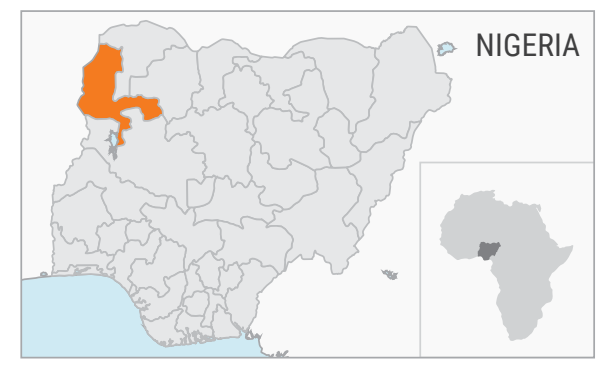

\section{Sakamakon binciken da aka yi a jihar ta Kebbi}

Binciken kan dabi'un matan ya gano cewa, muhimman abubuwa guda uku ne da za su inganta neman magani da kulawa daga kwararren ma'aikacin lafiya ga yaran da suka kamu da zazzabi. Adadin kowanne maki a cikin akwati shi ne maki kasa da tsaka-tsakin makin (kashi 50 daga cikin dari). Rabin kananan hukumomi sun samu makin fiye da hamsin (masu launin ja) kuma sauran kananan hukumomi sun samu maki fiye da hamsin (masu launin kore).

\section{Mata sun yarda cewa babu wanda ya fi cancanta a tuntuba a lokacin da yaro ya kamu da rashin lafiya illa ma'aikacin lafiya}

Kananan hukumomin da

ke dauke da launin ja

sune ke da kasa da

maki 99 daga

cikin dari. Sun

kuma yarda da

cewa ya dace

a tuntubi

ma'aikacin

lafiya a duk

lokacin da yaro ya

kamu da rasrashin lafiya.

Masu launin kore su ne

sama da haka.

\section{Mata sun yarda cewa kananan asibitoci \\ a unguwaninsu na iya ba da magani/kul- awa wa yaron da ba shi da lafiya}

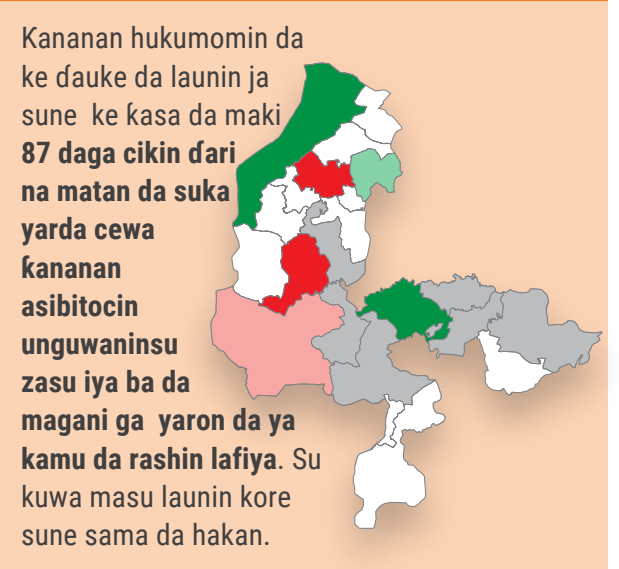

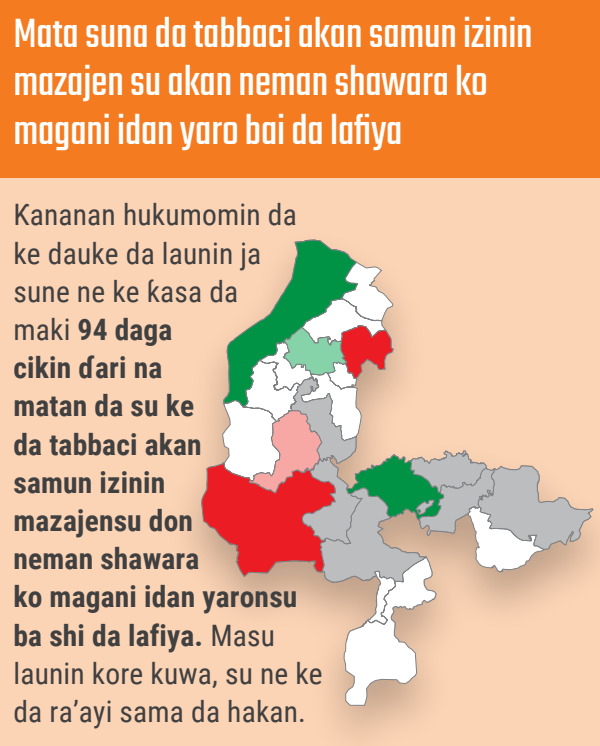

Karin bayani: Bangar da aka sanya wa launi mai haske sune ke dab da mataki na kasan ko saman tsakiya. Yankuna masu launi duhu-duhu, sun nesanta da na tsakiya, a inda yake nuni da kasa da kashi 25 daga cikin dari ko sama da kashi 75 daga cikin dari. Kananan hukumomi da ke dauke da launin fari ba sa cikin samfurin nazarin Saboda haka, babu bayani maki kamar yadda aka yi wa sauran. Kananan hukumomin da ke da launin ruwan toka-toka an sami karancin a lokacin da ake gudanar da binciken. Saboda haka, ba su isa a samu isheshen bayanin da ake nema a kansu ba.

\section{Ta ya ya ne za a yi amfani da wannan bayani don inganta neman maganin zazzabi da kulawa ga yara a yayin da suka kamu da rashin lafiya a jihar Kebbi?}

Tattaunawa da tambayoyi akan kalubalen da aka fuskanta aabubuwan da suke taimakawa wajen neman maganin zazzabin yara na iya samun mahanga akan yanda za a inganta taimako a waddanan bangarori ta hanyar yin tambayoyi kamar haka:

- Mene ne ya bambanta a shiyoyin kananan hukumomin da ke dauke da launin kore da zai iya bayyana nagartar tafyar da aikinsu a kananan asibitocin? Shin, ko kananan hukumomi masu dauke da launin kore suna samun karin tallafin kayan aiki daga waje da kuma samun horon inganta kwarewa wajen tafiyar da aikinsu da na wayar da kan al'umma?

- Mene ne irin bambancin da ke da akwai a kan kayayyakin asibitocin kananan hukumomi da aka samar da mata zasu zai iya alfaharin cewa suna cin moriyarsa? Shin ko kayayyakin tafiyar da aiki a asibitoci na taimakawa kuma ana kulawa da su kamar yadda ya kamata? Shin ma'aikatan lafiya na tafiyar da aikinsu yadda ya kamata tare da ba marasa lafiya kyawawan shawarwarin da suka dace?

- Mene ne zai bambanta al'ummomin da zasu iya bayar da cikakken bayanin irin gudumawar da kananan hukumomi ke bayar wa a fagen lura da lafiya? Shin, ko suna da jaruman shugabanni da nagartattun kwamitoci a kowanne yankin, ko ana bayar da dama ga 'yan yankin da su shigo domin bayar da gudumawa a kan harkar bunkasa dorewar kula da lafiyar jama'ar yankin?

- Wasu kalubale ne a cikin kananan hukumomi masu launin ja da suke hana mata neman magani daga ma'aikacin asibiti idan yaransu suka kamu da zazzabi? Ta ya ya ne shugabanni da sauran al'ummar yankin za su iya kawo karshen wadannan kalubalen?

- Shin kananan hukumomin masu launin kore na da abubuwan da ke karfafa wa mata a kan neman magani daga ma'aikacin lafiya idan yaransu na fama da zazzabi? Ta ya ya za su yi amfani da hakan a dace a yankuna masu karancin nema?

- Ta yay a za a inganta aikin ko bayanai zuwa ga al'ummomi da gidajen da ke cikin matukar bukatar ingantacen bayanai a kan muhimancin neman magani da kulawa?

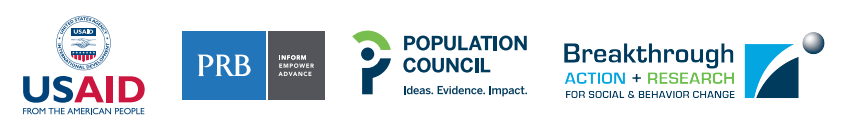

